\title{
Análisis biomecánico para confirmar el diagnóstico en neurorrehabilitación
}

\author{
de los Reyes-Guzmán, Ana ${ }^{a}$; López-Dolado, Elisab ${ }^{b}$ Pérez-Rizo, Enrique ${ }^{a}$; Lozano- \\ Berrio, Vicente ${ }^{a}$; Gil-Agudo, Ángel ${ }^{\mathrm{b}}$ y del-Ama, Antonio J. ${ }^{a}$ \\ aUnidad de Biomecánica y Ayudas Técnicas, Hospital Nacional de Parapléjicos (SESCAM), Toledo, \\ España,\{adlos;enriquep;vlozanob;ajdela\}@sescam.jccm.es \\ bServicio de Rehabilitación, Hospital Nacional de Parapléjicos (SESCAM), Toledo, España, \{elopez, \\ amgila\}@sescam.jccm.es
}

\begin{abstract}
In cervical spinal cord injured (SCI) patients, upper limbs strength is affected to a greater or lesser extent, producing dependence on the execution of ADLs. If the lesion is incomplete, the gait ability can be preserved. In this context, it is difficult to make a correct clinical diagnosis and the photogrammetry equipments constitute a tool of great value to determine with objectivity motor sequelae. The objective is to present the biomechanics methodology of upper and lower limbs applied to a case study. It is a 61year-old male patient who suffered an incomplete SCI of traumatic etiology that had previously suffered a traumatic brain injury. Through photogrammetry, the range of motion of the hip, knee and ankle joints were analyzed during the cycles of the gait and the shoulder, elbow and wrist joints, as well as a series of kinematic indices describing the ability and dexterity of the upper limb movement.

A very symmetrical gait pattern was obtained in both lower limbs. However, overall functionality and skill indices in both upper limbs present a high asymmetry between them. Biomechanical tools demonstrate aspects of motor control not easily visible with clinical tests and perfect diagnosis of complex cases.
\end{abstract}

Keywords:biomechanics, kinematics, kinetic, lower limb, upper limb, stroke, spinal cord injury.

\section{Resumen}

En los pacientes con lesión medular cervical (LMC) se ve comprometida en mayor o menor medida la fuerza de las extremidades superiores, lo que se traduce en dependencia para las AVDs. Si la lesión es incompleta, puede preservarse la capacidad de marcha. En este contexto, resulta dificil realizar un diagnóstico clínico correcto y los equipos de fotogrametría constituyen 
una herramienta de gran valor para objetivar las secuelas motoras. El objetivo es presentar la metodología biomecánica de miembros superiores e inferiores aplicada a un caso de estudio. Se trata de un paciente varón de 61 años que padeció una LMC incompleta de etiología traumática que, previamente, había sufrido un TCE. Mediante fotogrametría se analizaron los recorridos articulares de la cadera, rodilla y tobillo durante los ciclos de la marcha y del hombro, codo y muñeca, así como una serie de índices cinemáticos descriptores del movimiento del miembro superior.

Se obtuvo un patrón de marcha muy simétrico en ambos miembros inferiores. Sin embargo, la funcionalidad global y los indices de destreza en ambos miembros superiores presentaron una marcada asimetría entre ellos. Las herramientas biomecánicas evidencian aspectos del control motor no fácilmente visibles con los tests clínicos y que perfeccionan el diagnóstico de los casos complejos.

Palabras clave:biomecánica, cinemática, cinética, miembro inferior, miembro superior, ACV, Lesión medular.

\section{Introducción}

En los últimos años, el aumento de la esperanza de vida ha traído como consecuencia un incremento en el número de traumatismos del sistema nervioso central (SNC) en la población de más edad. A su vez, las secuelas neurológicas aumentan el riesgo de caídas y, por lo tanto, el riesgo de sufrir nuevos eventos traumáticos.

La afectación de la extremidad superior es una de las secuelas más habituales en las lesiones del SNC (Parker, 1986) y, en el caso de la lesión medular afecta a más del 50\% de los casos (Wyndaele, 1996).

En los pacientes con lesión medular cervical (LMC) se ve comprometida en mayor o menor medida la fuerza de las extremidades superiores, lo que se traduce en dependencia para las AVDs y pérdida de autonomía personal. Si la lesión es incompleta, puede preservarse la capacidad de marcha. Cuando hay secuelas sensitivomotoras previas, éstas ocultan los síntomas y signos de localización de la lesión medular y resulta difícil realizar un diagnóstico clínico correcto. Por este motivo, resulta necesaria la aplicación de elementos objetivos de medida y sistemas precisos de análisis de movimiento para describir con una mayor precisión y especificidad el movimiento humano.

En este contexto, los estudios biomecánicos son una herramienta de gran valor para objetivar la marcha humana y la ejecución de movimientos correspondientes a actividades 
funcionales de los miembros superiores. Aplicados al análisis de la marcha humana, su utilidad se centra, fundamentalmente, en servir de herramienta de valoración en pacientes que presentan un cuadro conocido para tomar decisiones clínicas y planificar su tratamiento, así como para evaluar posteriormente la eficacia de las medidas adoptadas. (Gil-Agudo, 2019).

En cuanto a los estudios para objetivar la función del miembro superior son numerosos los realizados en población sana (Namdari, 2012; Murgia, 2010; Aizawa, 2010; van Andel, 2008; Petuskey, 2007; Murphy, 2006; Magermans, 2005). Posteriormente, estos estudios se fueron extendiendo para cuantificar objetivamente la capacidad funcional y rangos de movimiento articular en presencia de patologías neurológicas. En cuanto a estas enfermedades, las estudiadas con más frecuencia han sido el accidente cerebrovascular (ACV) (Kim, 2014; Murphy, 2013; Murphy, 2011; Osu, 2011; Lang, 2006) y la Parálisis Cerebral (Klotz, 2013; Butler, 2010). Todavía los estudios aplicados a la LM son escasos frente a los realizados en otras patologías. No obstante, existe un trabajo previo que revisa los estudios cinemáticos del miembro superior realizados en pacientes con LM (Mateo, 2015).

Actualmente, en nuestro grupo, ambas pruebas biomecánicas están bien consolidadas y establecidas para su aplicación en pacientes con Lesión Medular o cualquier otra patología neurológica que entre sus secuelas produce trastornos de la marcha humana y afectación de la función de los miembros superiores. Sin embargo, no se tiene evidencia de estudios previos que apliquen ambas pruebas biomecánicas conjuntamente a la muestra analizada con el objetivo de ayudar al diagnóstico clínico. Así, el objetivo de este trabajo es presentar la metodología biomecánica de miembros superiores e inferiores aplicada a un caso de estudio.

\section{Métodos}

Se trata de un estudio descriptivo, experimental de estudio de un caso, el de un varón de 61 años que sufre una lesión medular cervical traumática aguda incompleta al caerse de una bicicleta. El paciente había sufrido cinco años antes un traumatismo craneoencefálico con resultado de hemorragia intraparenquimatosa parietal izda que le había dejado como secuela una monoparesia espástica derecha mínima. Las pruebas de imagen tras la caída evidenciaron una fractura de la lámina izda C6 sin desplazamiento vertebral ni estenosis de canal con contusión medular $\mathrm{C} 4$ y afectación de la musculatura cervical posterior y los ligamentos interespinosos desde $\mathrm{C} 2-\mathrm{C} 5$. Se desestima tratamiento quirúrgico y se le remite a un hospital monográfico de lesión medular para que realice un programa de rehabilitación integral. A su llegada a dicho centro presentaba una lesión medular de nivel C4 incompleto sensitivomotor ASIA D que no se ajustaba bien a ninguno de los síndromes clínicos de 
lesión incompleta, lo que dificultaba establecer el pronóstico funcional, pues tanto las excalas clínicas como los estudios habituales de neuroimagen y neurofisiológicos estaban interferidos por las secuelas sobre el miembro superior derecho del traumatismo craneoencefálico antiguo. Se decidió analizar el movimiento de los miembros superiores y la marcha del paciente con herramientas biomecánicas, con el objetivo de obtener datos objetivos y cuantitativos del control motor en este paciente que pudieran enriquecer el diagnóstico clínico.

Ambas pruebas biomecánicas se realizaron en el período subagudo aunque no simultáneamente. Se realizaron instrumentando al paciente con el equipo de fotogrametría Codamotion (Charnwood Dynamics, Ltd, UK) basado en marcadores activos, muestreando a una frecuencia de $200 \mathrm{~Hz}$. Adicionalmente, para el análisis de la marcha se utilizaron dos plataformas dinamométricas Kistler para registrar de forma simultánea la fuerza de reacción durante la fase de apoyo en ambos miembros inferiores.

\subsection{Análisis biomecánico de la marcha}

Para la realización del estudio biomecánico de la marcha se posicionaron 22 marcadores activos los miembros inferiores y en la pelvis, siguiendo el modelo descrito previamente por otros autores (Kadaba, 1989). Los marcadores se posicionaron en las marcas anatómicas descritas en nuestro trabajo previo (Gil-Agudo, 2009) para analizar los recorridos articulares de la cadera, rodilla y tobillo durante los ciclos de la marcha. A partir de las posiciones de los marcadores se calcularon los desplazamientos de las siguientes referencias anatómicas: espinas ilíacas anterosuperiores, espinas ilíacas posterosuperiores, epicondilos medial y lateral, ambos maléolos, calcáneo y $5^{\mathrm{a}}$ articulación metacarpofalágica.

Una vez instrumentado el paciente, éste se situó al inicio del pasillo de la marcha. Antes de iniciar la adquisición de pruebas el sujeto realizó varias pasadas por el pasillo de macha a modo de adaptación, con su velocidad de marcha habitual. Posteriormente, se registraron una serie de pasadas hasta lograr un mínimo de cinco pruebas correctas (en función del pisado completo sobre las plataformas).

\subsubsection{Procesamiento de los datos y variables analizadas}

Una vez terminada la adquisición, se procede al análisis de los resultados mediante la utilización de un modelo biomecánico de ambos miembros inferiores escalado a las dimensiones antropométricas del paciente (Fig.1A).

En cuanto a la cinemática de los miembros inferiores se analizaron los valores máximos y mínimos tanto de la fase de apoyo como de la de balanceo, el momento del ciclo en que 
aparecen (expresado en \% del ciclo de marcha), la amplitud articular (diferencia entre el valor máximo y el mínimo de todo el ciclo independientemente de si se sitúa en el apoyo o en el balanceo) así como, los siguientes valores críticos: contacto inicial y despegue de dedos. La recogida de datos se efectuó de todas las articulaciones, cadera, rodilla y tobillo, en cada uno de sus recorridos articulares posibles.

Asimismo, se analizaron los parámetros espaciotemporales de la marcha como la velocidad de marcha $(\mathrm{m} / \mathrm{s})$, duración del ciclo de marcha $(\mathrm{s})$, longitud del ciclo $(\mathrm{cm})$, duración del paso (s), cadencia (pasos/min), duración de la fase de apoyo y de oscilación (expresado en $\%$ de la duración del ciclo de marcha).

\subsection{Análisis cinemático del miembro superior}

Los estudios cinemáticos del miembro superior se realizan simulando la AVD de beber de un vaso. El setup experimental y la configuración del laboratorio se describe en detalle en un trabajo previo nuestro realizado en población sana y en pacientes con LMC (de los Reyes-Guzmán, 2010). En ese estudio, todos los participantes eran diestros y realizaron el gesto de beber con el miembro superior derecho a velocidad libre.

Sin embargo, en este trabajo se realiza el estudio cinemático en ambos miembros superiores aunque no de forma simultánea, debido a la complejidad del estudio en cuanto a instrumentación. Por tanto, se realizan en sesiones diferentes, muy próximas entre sí para asegurar que no se producen cambios en la capacidad funcional del paciente.

\subsubsection{Sesiones experimentales}

Las sesiones experimentales sobre cada miembro superior se realizaron una sola vez. El registro del movimiento se realizó con el mismo equipo de fotogrametría que el utilizado para el análisis de la marcha humana.

En cada sesión se instrumentó el tronco y brazo cuyo movimiento se analiza con 21 marcadores de fotogrametría: 8 se ubicaron en prominencias óseas para formar el modelo biomecánico y los otros 13 se localizaron en clusters de marcadores para conocer la posición y orientación de los segmentos corporales durante el movimiento. Estos 13 marcadores se reparten de la siguiente forma: 9 repartidos en clusters de 3 marcadores ubicados en tronco, brazo y antebrazo; 3 marcadores en la parte dorsal de la mano sobre la piel y uno adicional en el tronco.

Una vez instrumentado el paciente, la actividad analizada consistía en alcanzar y agarrar el vaso desde la posición de inicio, llevar el vaso a la boca, beber (equivalente a un trago), llevar el vaso al área marcada en la mesa y volver a la posición de inicio. Antes de registrar 
el movimiento todos los sujetos realizaron la actividad dos veces a modo de aclimatación. Se capturaron y analizaron 5 registros válidos.

\subsubsection{Procesamiento de los datos y variables analizadas}

Para el procesamiento de los datos se utilizó el software Visual3D (C-Motion, Inc, USA) en el que se implementó un programa de tratamiento y procesamiento de la señal encargado de obtener las señales del movimiento de las distintas articulaciones involucradas (hombro, codo y muñeca). La frecuencia de muestreo era de $200 \mathrm{~Hz}$. Se analizaron cinco registros y la media de las cinco medidas fue el valor final para cada una de las variables analizadas.

Para el análisis cinemático del miembro superior derecho, se implementó en Visual3D un modelo biomecánico de 9 grados de libertad: 2 en el tronco, 3 en el hombro, 2 en el codo y 2 en el carpo. Para cada segmento del modelo se definió un sistema de coordenadas local siguiendo las recomendaciones de la Sociedad Internacional de Biomecánica (ISB) (Wu, 2005) (Fig.1B). De la mismaforma se implementó el modelo para analizar el movimiento del miembro superior izquierdo.
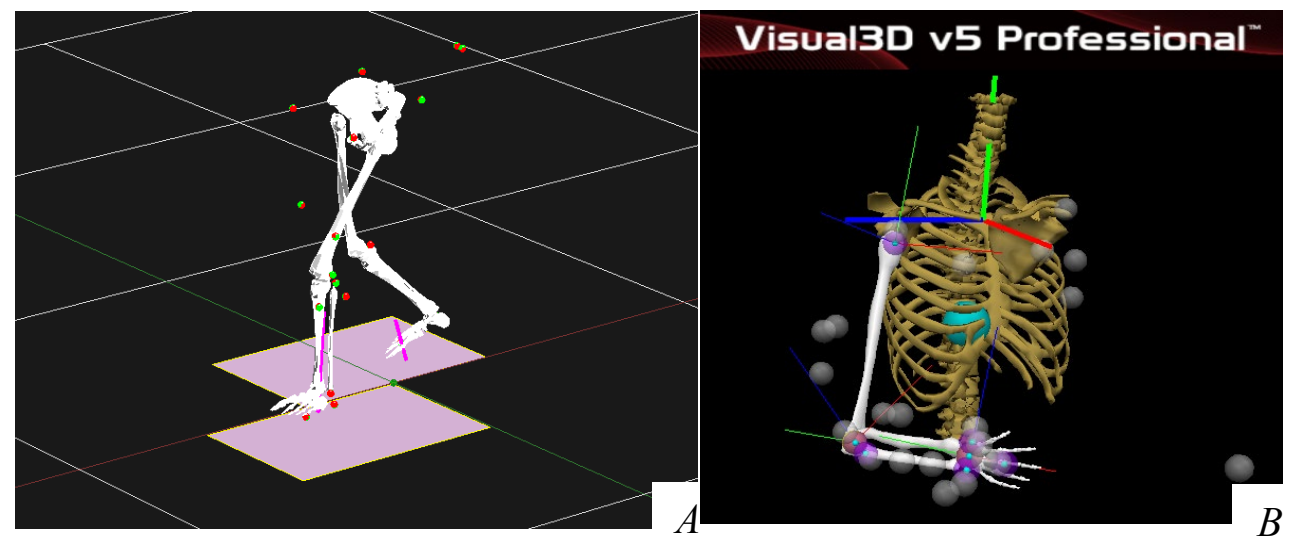

Fig.1: A) Modelo biomecánico de los miembros inferiores escalado a las dimensiones antropométricas del paciente (2016). B) Modelo biomecánico del miembro superior derecho escalado a las dimensiones antropométricas del paciente (2016).

En cada uno de los registros se identificó un ciclo completo de la actividad de beber. Se consideró que el inicio del ciclo se correspondía con el inicio del desplazamiento del marcador situado en la cabeza del tercer metacarpiano y el final de dicho ciclo cuando ese marcador se volvía a situar en la posición inicial tras realizar la actividad. Basándonos en estudios previos (Murphy, 2006; de los Reyes-Guzmán, 2010), se establecieron una serie de fases para facilitar el análisis de la actividad: alcance (incluye coger el vaso), transporte proximal, beber, transporte distal (incluye soltar el vaso) y retorno a inicio. 
Una vez recogidos y analizados los registros, a partir de los recorridos articulares y variables como la posición y velocidad de la mano, medida a partir del marcador en la cabeza del tercer metacarpiano, se computaron una serie de índices cinemáticos descriptores del movimiento, relacionados con destreza y habilidad global del miembro superior, como son precisión, agilidad, eficiencia, suavidad y coordinación (de los ReyesGuzmán, 2017; de los Reyes-Guzmán, 2016).

\section{Resultados}

A continuación se muestran la evolución neurológica y funcional del paciente y los resultados obtenidos en ambas pruebas biomecánicas realizadas.

\subsection{Evolución neurológica}

A su ingreso, el paciente presentaba una lesión medular cervical de nivel C4 incompleta sensitivomotora grado D según la escala ASIA (Kirshblum, 2011) (Fig 2A y 2B) y al alta a los seis meses de evolución, una vez concluido el programa rehabilitador intensivo, mostraba una clara mejoría global, tanto sensitiva como motora y funcional (Fig. 2 C, D y E respectivamente).
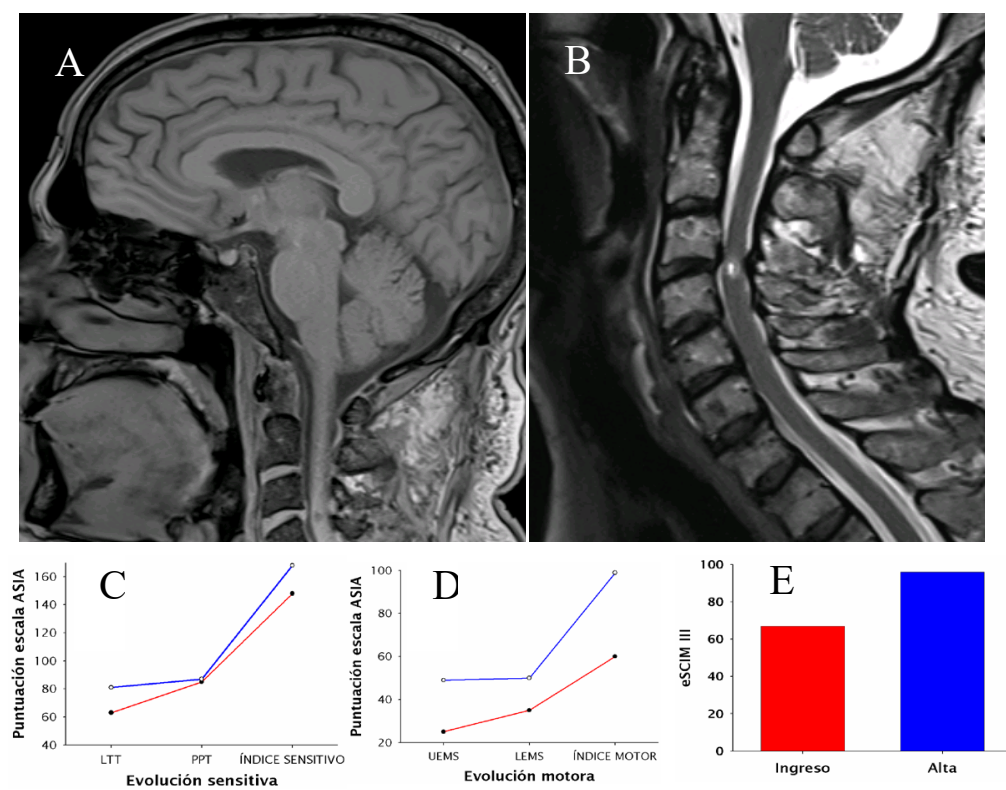

Fig.2: A) Corte sagital en T1 de resonancia magnética craneal a los 6 meses de lesión, donde se comprueba la normalidad del encéfalo y la lesión medular en C4. B) Corte sagital en T2 de resonancia magnética de columna cervical. $\boldsymbol{C}, \boldsymbol{D} \boldsymbol{y} \boldsymbol{E})$ Evolución de la lesión medular desde un punto de vista sensitivo, motor y funcional, respectivamente. 


\subsection{Análisis biomecánico de la marcha}

Parámetros temporales: Se detectaron las habituales alteraciones en la marcha de personas con lesión medular, es decir, disminución de la velocidad, de las longitudes de ciclo y de paso, de la frecuencia de ciclos/minuto y de la cadencia. Por el contrario, aumento de la duración de ciclo y de paso y el porcentaje de duración de la fase de apoyo (tabla 2).

Cinemática articular: En el plano transversal de la pelvis existía una rotación pélvica con elevación de la hemipelvis derecha. En el plano frontal, se observaba la cadera derecha en abducción durante la fase de apoyo, probablemente para aumentar la base de apoyo y ganar estabilidad en el apoyo monopodal. La cadera derecha se mantenía en rotación externa a lo largo del ciclo. El pico normal de flexión de rodilla en la fase de oscilación de la marcha estaba disminuido en ambas rodillas, que se posicionaban en rotación externa siempre en el plano transversal. En el tobillo, se observaba que el pico normal de flexión plantar en el despegue de dedos, necesario para el adecuado despegue del pie y avance del miembro inferior durante la fase de oscilación, estaba disminuido. Por último, ambos pies se mantenían en abducción durante el ciclo de la marcha (tabla 3).

Tabla 1. Resultados de los parámetros espaciotemorales de ambas extremedades inferiores del paciente y de la base de datos de normalidad.

\begin{tabular}{ccccc}
\hline $\begin{array}{c}\text { Parámetros } \\
\text { espacio-temporales }\end{array}$ & Izquierdo & Derecho & Normalidad & $\begin{array}{c}\text { Desviación } \\
\text { estándar }\end{array}$ \\
\hline Velocidad (m/s) & $0.77 \pm 0.03$ & $0.7 \pm 0.03$ & 1.27 & 0.12 \\
Longitud ciclo (m) & $1 \pm 0.03$ & $0.95 \pm 0.02$ & 1.33 & 0.08 \\
Duración Ciclo (s) & $1.31 \pm 0.03$ & $1.35 \pm 0.04$ & 1.05 & 0.08 \\
Ciclos / Minuto & $45.9 \pm 1.08$ & $44.58 \pm 1.25$ & 57.33 & 4.16 \\
Longitud paso (m) & $0.52 \pm 0.01$ & $0.49 \pm 0.03$ & 0.67 & 0.04 \\
Duración paso (s) & $0.65 \pm 0.02$ & $0.67 \pm 0.02$ & 0.53 & 0.04 \\
Pasos / Minuto & $92.9 \pm 2.91$ & $90.4 \pm 3.23$ & 114.65 & 8.30 \\
Porcentaje & $64.95 \pm 1.38$ & $67.45 \pm 0.96$ & 63.13 & 1.62 \\
apoyo(\%) & & $0.46 \pm 0.02$ & 0.39 & 0.03 \\
Apoyo unipodal (s) & $0.44 \pm 0.02$ & $0 \pm 0$ & 0.14 & 0.02 \\
Apoyo bipodal (s) & $0.22 \pm 0.01$ & & & \\
Contacto inicial de & $50.65 \pm 1.2$ & $50.75 \pm 1.11$ & & \\
pie contralateral (\%) & & & & \\
\hline
\end{tabular}


de los Reyes-Guzmán, A.; López-Dolado, E.; Pérez-Rizo, E.; Lozano-Berrio, V.; Gil-Agudo, A. y del-

Tabla 2. Resultados de las variables cinemáticas articulares (expresadas en ${ }^{0}$ ) del paciente y de la base de datos de normalidad.

\begin{tabular}{|c|c|c|c|c|}
\hline $\begin{array}{c}\text { Ángulos } \\
\text { articulares plano } \\
\text { sagital }\left(^{\circ}\right)\end{array}$ & Izquierdo. & Derecho & Normalidad & $\begin{array}{c}\text { Desviación } \\
\text { estándar }\end{array}$ \\
\hline Rango de cadera & $35.93 \pm 0.3$ & $32.35 \pm 1.42$ & 42.30 & 4.16 \\
\hline Máximo cadera & $\begin{array}{c}37.83 \pm \\
37.83\end{array}$ & $35.57 \pm 0.41$ & 39.92 & 7.26 \\
\hline Mínimo cadera & $1.9 \pm 0.43$ & $3.22 \pm 1.18$ & -0.39 & 7.26 \\
\hline Rango de rodilla & $53.57 \pm 1.7$ & $47.05 \pm 1.14$ & 61.84 & 3.46 \\
\hline Máximo rodilla & $53.17 \pm 1.22$ & $49.33 \pm 1.87$ & 63.03 & 3.50 \\
\hline Mínimo rodilla & $-0.4 \pm 0.83$ & $2.28 \pm 1.02$ & 1.87 & 3.81 \\
\hline Rango de tobillo & $17.02 \pm 1.95$ & $16.9 \pm 0.84$ & 31.57 & 5.42 \\
\hline Máximo tobillo & $7.64 \pm 1.56$ & $9.14 \pm 1.1$ & 12.40 & 2.47 \\
\hline Mínimo tobillo & $-9.38 \pm 0.64$ & $-7.76 \pm 0.73$ & -17.21 & 4.81 \\
\hline Rango de cadera & $35.93 \pm 0.3$ & $32.35 \pm 1.42$ & 42.30 & 4.16 \\
\hline
\end{tabular}

\subsection{Análisis cinemático del miembro superior}

Los resultados relativos al estudio biomecánico del miembro superior derecho e izquierdo se representan en la tabla 3 , diferenciados según se trate de rangos de movimiento articular, variables temporales e índices cinemáticos descriptores del movimiento.

\subsubsection{Rangos de movimiento articular (ROM)}

En el análisis del miembro superior derecho se obtuvo un ROM total de $82^{\circ}$ en el hombro, siendo $42.83^{\circ}$ en el movimiento de flexión-extensión, $25.14^{\circ}$ en el movimiento de abducción-adducción y $14.03^{\circ}$ en rotación externa-interna; en la articulación del codo se obtuvo un total de $131.15^{\circ}$ de los que $90.46^{\circ}$ corresponden al movimiento de flexiónextensión y 40.69 a la pronación-supinación del antebrazo; en cuanto a la muñeca se midieron $14.19^{\circ}$ en el movimiento de flexión-extensión. 
Tabla 3. Resultados de las variables cinemáticas en ambas evaluaciones realizadas al paciente

\begin{tabular}{ccc}
\hline $\begin{array}{c}\text { Variables } \\
\text { cinemáticas }\end{array}$ & $\begin{array}{c}\text { Miembro superior } \\
\text { derecho }\end{array}$ & $\begin{array}{c}\text { Miembro superior } \\
\text { izquierdo }\end{array}$ \\
\hline ROM ( ${ }^{\text {) }}$ & & \\
Flex-ext hombro & 42.83 & 36.08 \\
Abd-add hombro & 25.14 & 15.85 \\
Rot. Ext-Int & 14.03 & 19.53 \\
hombro & & 74.57 \\
Flex-ext codo & 90.46 & 45.02 \\
Pron-sup codo & 40.69 & 76.25 \\
Flex-ext muñeca & 14.19 & 8.23 \\
Tiempo (s) & 8.10 & 0.76 \\
Vel. max (m/s) & 0.70 & 0.24 \\
Vel. med (m/s) & 0.23 & 104.78 \\
Índices \\
cinemáticos (\%)
\end{tabular}

En cuanto al miembro superior izquierdo se obtuvo un ROM total de $71.46^{\circ}$ en el hombro, siendo $36.08^{\circ}$ en el movimiento de flexión-extensión, $15.85^{\circ}$ en el movimiento de abducción-adducción y $19.53^{\circ}$ en rotación externa-interna; en la articulación del codo se obtuvo un total de $119.59^{\circ}$ de los que $74.57^{\circ}$ corresponden al movimiento de flexiónextensión y 45.02 a la pronación-supinación del antebrazo; en cuanto a la muñeca se midieron $76.25^{\circ}$ en el movimiento de flexión-extensión. 


\subsubsection{Variables temporales}

En cuanto a la duración del ciclo completo de movimiento, los resultados fueron muy parecidos en ambos miembros superiores: $8.10 \mathrm{~s}$ en el caso del miembro superior derecho y $8.23 \mathrm{~s}$ en el miembro superior izquierdo. Lo mismo ocurrió en cuanto a las velocidades de movimiento (Tabla 3).

\subsection{3. Índices cinemáticos}

Los resultados de los índices cinemáticos se representan en \%, relativos al patrón de referencia formado por un grupo de personas sanas (de los Reyes-Guzmán, 2016; de los Reyes Guzmán, 2017). Se ha obtenido en el miembro superior izquierdo un menor desempeño en los índices Precisión (64.92) y Agilidad (62.79) que aquellos en el miembro superior derecho (91.68 y 88.90, respectivamente). Sin embargo en el izquierdo se ha obtenido un mejor desempeño en cuanto a los índices suavidad y coordinación, que superan la puntuación del patrón de referencia. Estos resultados se pueden observar de forma más sencilla en la Fig.3.

\section{ÍNDICES DE VALORACIÓN FUNCIONAL}

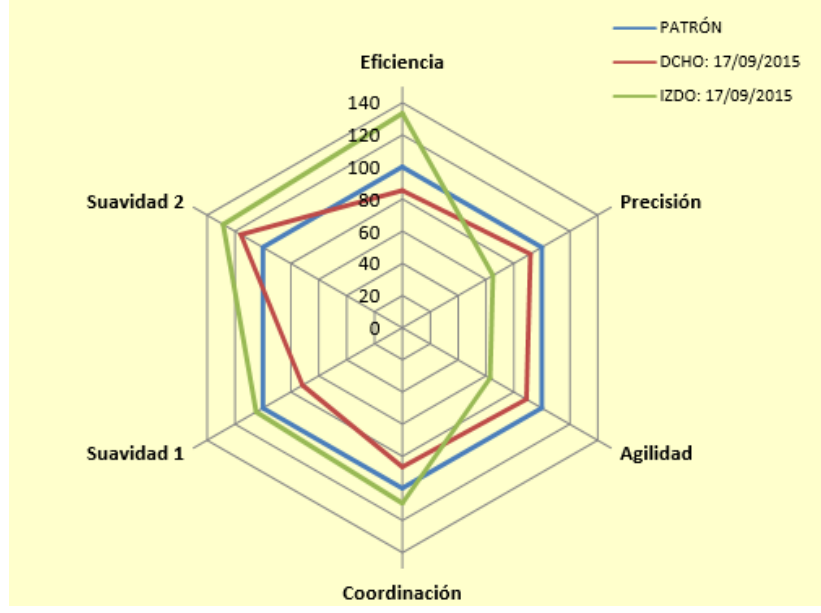

Fig. 3. Representación gráfica de los índices cinemáticos relativos a las evaluaciones del miembro superior derecho (rojo) e izquierdo (verde) (2015).

\section{Discusión}

El paciente que se presenta había sufrido dos lesiones traumáticas del sistema nervioso: un primer traumatismo craneoencefálico que le dejó como secuela una monoparesia espástica 
derecha cinco años atrás y una lesión medular cervical incompleta aguda. Su edad y el mecanismo de lesión apuntaban hacia el síndrome centromedular o de Schneider (Schneider, 1958). Sin embargo, la neuroimagen inicial mostraba un gran componente edematoso y no se ajustaba a la localización centromedular y el paciente había experimentado una clara progresión hacia la mejoría neurológica global, pero ésta había sido mayor en los miembros superiores que en los inferiores (Fig. 2C y 2D), justo al contrario de lo esperado en el síndrome de Schneider. Además, a pesar de que la exploración clínica de la fuerza voluntaria residual de los brazos era bastante simétrica, el paciente refería grandes diferencias en la ejecución motora entre el brazo derecho y el izquierdo.

Dado que la práctica totalidad de la recuperación funcional en los miembros superiores tras una lesión medular cervical sucede en los seis primeros meses en el síndrome centromedular (McKinley, 2007), se decidió evaluar la calidad de la función motriz mediante estudios biomecánicos, que mostraron: 1) que la función locomotriz de ambas piernas era simétrica y se encontraba más próxima a la normalidad que la de los brazos; 2) que ambos miembros superiores estaban severamente afectados pero de manera asimétrica como refería el paciente; 3) que las diferencias entre los miembros superiores se debían la coincidencia en el brazo derecho de las secuelas del traumatismo craneoencefálico antiguo y de la lesión medular cervical actual y se expresaban y cuantificaban consistentemente gracias a los índices cinemáticos. Se concluyó entonces que el diagnóstico más probable del paciente era un síndrome centromedular C4 ASIA D y que la función de ambos brazos, en consecuencia, tenía un pronóstico de recuperación funcional en adelante muy restringido. La resonancia magnética de control realizada a los 6 meses mostró la mielopatía centromedular (Fig. 2A y 2B) y las exploraciones sucesivas al año y a los dos años evidenciaron estancamiento de la manipulación con mejoría discreta en la marcha.

Una limitación del presente trabajo es que la realización de ambos estudios biomecánicos fue secuencial y no simultánea, si bien entre la realización del estudio de marcha y el de los miembros superiores se realizaron en la misma semana. Existe muy pocos trabajos cuya metodología utilice simultáneamente el análisis biomecánico de los miembros superiores y de la marcha. Sin duda, la instrumentación necesaria para aplicar esta doble metodología requiere largos tiempos de intrumentación y probablemente la interpretación de los resultados obtenidos no sea sencilla. Sin embargo, el presente trabajo sugiere que contar con ella podría ser de gran ayuda para mejorar la potencia diagnóstica y para entender mejor la motricidad residual global del paciente que sufre una lesión neurológica compleja. 


\section{Conclusión}

El presente trabajo pretende poner de relieve la capacidad de los estudios biomecánicos para exclarecer dudas diagnósticas o pronósticas de la práctica clínica diaria en neurorrehabilitación gracias a que aportan datos objetivos y cuantitativos del movimiento.

Metodológicamente, se trata de estudios que poseen una elevada complejidad en cuanto a la instrumentación necesaria en el paciente y al procedimiento de análisis de los datos obtenidos. Aun así, hoy en día, siguen siendo de especial interés por tratarse de la tecnología gold-standard en análisis de movimiento dentro del entorno clínico.

\section{Referencias}

AIZAWA, J., MASUDA, T., KOYAMA, T., KOJIISOKZAKI, K.N., OKAWA, A., MORITA, S., (2010). "Three dimensional motion of the upper extremity joints during various activities of daily living”. J Biomech, vol. 43, pp. 2915-2922.

BUTLER, E.E., LADD, A.L., LOUIE, S.A., LAMONT, L.E., WONG, W., ROSE, J., (2010). "Temporalspatial parameters of the upper limb during a reach \& grasp cycle for children". Gait Posture, vol. 32, pp. 301-306.

DE LOS REYES-GUZMÁN, A., GIL-AGUDO, A., PEÑASCO-MARTÍN, B., SOLÍS-MOZOS, M., DEL AMA-ESPINOSA, A., y PÉREZ-RIZO, E. (2010). "Kinematic analysis of the daily activity of drinking from a glass in a population with cervical spinal cord injury". Journal of neuroengineering and rehabilitation, 7(1), 41.

DE LOS REYES-GUZMÁN, A., DIMBWADYO-TERRER, I., PÉREZ-NOMBELA, S., MONASTERIO-HUELIN, F., TORRICELLI, D., PONS, J.L., y GIL-AGUDO, A. (2016). "Novel kinematic indices for quantifying movement agility and smoothness after cervical Spinal Cord Injury". NeuroRehabilitation, 38(2), 199-209.

DE LOS REYES-GUZMÁN, A., DIMBWADYO-TERRER, I., PÉREZ-NOMBELA, S., MONASTERIO-HUELIN, F., TORRICELLI, D., PONS, J.L., y GIL-AGUDO, A. (2017). "Novel kinematic indices for quantifying upper limb ability and dexterity after cervical spinal cord injury". Medical \& biological engineering \& computing, 55(5), 833-844.

GIL-AGUdO, A., PÉREZ-RIZO, E., DEL AMA-ESPINOSA, A., CRESPO-RUIZ, B., PÉREZNOMBELA, S., Sánchez-Ramos A., (2009). "Comparative biomechanical gait analysis of patients with central cord syndrome walking with one and two crutches". Clin Biomech (Bristol Avon) 24:551557.

GIL-AGUDO (2019). "Protocolo de valoración biomecánica de las alteraciones de la marcha". Medicine, 12(75):4462-6.

KADABA, M.P., RAMAKRISHNAN, H.K., WOOTTEN, M.E., GAINEY, J., GORTON, G., COCHRAN, G.V. (1989). "Repeatability of kinematic, kinetic, and electromyographic data in normal adult gait". J Orthop Res; 7(6): 849-860. 
KIM, K., SONG, W.K., LEE, J., LEE, H.Y., PARK, D.S., KO, B.W., KIM, J., (2014). "Kinematic analysis of upper extremity movement during drinking in hemiplegic subjects", Clin Biomech, doi: 10.1016/j.clinbiomech.2013.12.013.

KIRSHBLUM S.C., BURNS S.P., BIERING-SORENSEN F., DONOVAN W., GRAVES D.E., JHA A., JOHANSEN M., JONES L., KRASSIOUKOV A., MULCAHEY MJ, SCHMIDT-READ M., WARING W. (2011). "International standards for neurological classification of spinal cord injury (Revised 2011)". Journal of Spinal Cord Medicine, 34 (6): 535-46.

KLOTZ, M.C.M., KOST, L., BRAATZ, F., EWERBECK, V., HEITZMANN, D., GANTZ, S., DREHER, T., WOLF, S.I., (2013). "Motion capture of the upper extremity during activities of daily living in patients with spastic hemiplegic cerebral palsy". Gait Posture, 38,pp. 148-152.

LANG, C.E., WAGNER, J.M., EDWARDS, D.F., SAHMANN, S.A., DROMERICK, A.W., (2006). "Recovery of grasp versus reach in people with hemiparesis poststroke".Neurorehabil Neural Repair, vol. 20, pp. 444-454.

MAGERMANS, D., CHADWICK, E., VEEGER, H., VAN DER HELM, F., (2005). "Requirements for upper extremity motions during activities of daily living". Clin Biomech (Bristol, Avon), vol. 20, pp. 591-599.

MATEO, S., ROBY-BRAMI, A., REILLY, K.T., ROSSETTI, Y., COLLET, C., y RODE, G. (2015). "Upper limb kinematics after cervical spinal cord injury: a review". Journal of neuroengineering and rehabilitation, 12(1), 9 .

MCKINLEY, W., SANTOS, K., MEADE, M., BROOKE K. (2007). "Incidence and Outcomes of Spinal Cord Injury Clinical Syndromes”. J Spinal Cord Med. 2007;30:215-224

MURGIA, A., KYBERD, P., BARNHILLl, T., (2010). "The use of kinematic and parametric information to highlight lack of movement and compensation in the upper extremities during activities of daily living". Gait \& Posture, vol. 31, pp. 300-306.

MURPHY, M.A., WILLEN, C., SUNNERHAGEN, K.S., (2013). "Responsiveness of upper extremity kinematic measures and clinical improvement during the first three months after stroke". Neurorehabil Neural Repair, vol. 27, p. 844.

MURPHY, M.A., WILLEN, C., SUNNERHAGEN, K.S., (2011). "Kinematic variables quantifying upper extremity performance after stroke during reaching and drinking from a glass". Neurorehabil Neural Repair, vol. 25(1), pp. 71-80.

MURPHY, M.A., SUNNERHAGEN, K.S., JOHNELS, B., y WILLÉN, C. (2006). "Threedimensional kinematic motion analysis of a daily activity drinking from a glass: a pilot study". Journal of neuroengineering and rehabilitation, 3(1), 18.

NAMDARI, S., YAGNIK, G., EBAUGH, D.D., NAGDA, S., RAMSEY, M.L., WILLIAMS Jr, G.R., MEHTA, S.(2012). "Defining functional shoulder range of motion for activities of daily living". Journal of Shoulder and Elbow Surgery, vol. 21, no. 9, pp. 1177-1183.

OSU, R., OTA, K., FUJIWARA, T., OTAKA, Y., KAWATO M., LIU, M., (2011). "Quantifying the quality of hand movement in stroke patients through three-dimensional curvature". J Neuroeng rehabil, 8:62. 
PARKER, V.M., WADE, D.T., y HEWER, R.L. (1986). "Loss of arm function after stroke: measurement, frequency, and recovery”. International rehabilitation medicine, 8(2), 69-73.

PETUSKEY, K., BAGLEY, A., ABDALA, E., JAMES, M., RAB, G., (2007). "Upper extremity kinematics during functional activities: Three-dimensional studies in a normal pediatric population". Gait Posture, vol. 25, pp. 573-579.

SCHNEIDER R, THOMPSON J, BEBIN J (1958) "The syndrome of acute central cervical spinal cord Injury". J Neurol Neurosurg Psychiat 21:216-227.

VAN ANDEL, C.J., WOLTERBEEK, N., DOORENBOSCH, C.A., VEEGER, D.H., HARLAAR, J., (2008). "Complete 3D kinematics of upper extremity functional tasks". Gait Posture, vol. 27, pp. 120- 127.

WU, G., VAN DER HELM, F.C., VEEGER, H.D., MAKHSOUS, M., VAN ROY, P., ANGLIN, C. y WERNER, F.W. (2005). "ISB recommendation on definitions of joint coordinate systems of various joints for the reporting of human joint motion-Part II: shoulder, elbow, wrist and hand". Journal of biomechanics, 38(5), 981-992.

WYNDAELE, M., y WYNDAELE, J.J. (2006). "Incidence, prevalence and epidemiology of spinal cord injury: what learns a worldwide literature survey?". Spinal cord, 44(9), 523. 